\title{
Publisher's Note: Dynamics of filaments and membranes in a viscous fluid [Rev. Mod. Phys. 82, 1607 (2010)]
}

Thomas R. Powers

(Published 11 June 2010)

DOI: $10.1103 /$ RevModPhys.82.1945

PACS number(s): 02.40.Hw, 99.10.Fg

This paper was published online on 19 May 2010 with typographical errors on page 1608 and in the reference list. On page 1608, left-hand column, the fourth line should read as “...(Green and Zerna, 1968)...”; in the reference list, the fifty-second reference should read as "Green, A. E., and W. Zerna, 1968, Theoretical Elasticity, 2nd ed. (Dover, Mineola, NY)." The paper has been corrected as of 3 June 2010. The text and reference are correct in the printed version of the journal. 